\title{
Strong Exponential Attractors for Weakly Damped Semilinear Wave Equations
}

\author{
Cuncai Liu, Fengjuan Meng, and Chang Zhang \\ School of Mathematics and Physics, Jiangsu University of Technology, Changzhou 213001, China \\ Correspondence should be addressed to Chang Zhang; chzhnju@126.com
}

Received 4 May 2020; Accepted 10 August 2020; Published 1 October 2020

Academic Editor: Ali Jaballah

Copyright (c) 2020 Cuncai Liu et al. This is an open access article distributed under the Creative Commons Attribution License, which permits unrestricted use, distribution, and reproduction in any medium, provided the original work is properly cited.

In this paper, we investigate the longtime dynamics for the damped wave equation in a bounded smooth domain of $\mathbb{R}^{3}$. The exponential attractor is investigated in a strong energy space for the case of subquintic nonlinearity, which is based on the recent extension of the Strichartz estimate for the case of a bounded domain. The results obtained complete some previous works.

\section{Introduction}

Let $\Omega \subset \mathbb{R}^{3}$ be a bounded domain with smooth boundary. Given $\gamma>0$, we consider the following weakly damped wave equation:

$$
\begin{cases}u_{t t}+\gamma u_{t}-\Delta u+f(u)=g, & x \in \Omega, t>0, \\ u(x, 0)=u_{0}, u_{t}(x, 0)=u_{1}, & x \in \Omega, \\ u(x, t)=0, & x \in \partial \Omega, t>0 .\end{cases}
$$

Here, $g \in L^{2}(\Omega)$ is independent of time and the nonlinear function $f \in C^{3}(\mathbb{R})$ with $f(0)=0$ and satisfies the following conditions:

$$
\begin{aligned}
&\left|f^{\prime \prime \prime}(s)\right| \leq c_{1}\left(1+|s|^{p-3}\right), \\
& \liminf _{|s| \longrightarrow \infty} \frac{f(s)}{s}>-\lambda_{1},
\end{aligned}
$$

where $3 \leq p<5$ and $\lambda_{1}>0$ is the first eigenvalue of $-\Delta$ on $\Omega$ with Dirichlet boundary condition.

Denote by $\langle\cdot, \cdot\rangle$ and $\|\cdot\|$ the inner product and norm in $L^{2}(\Omega)$. For the sake of convenience, we define Hilbert spaces $\mathscr{H}=H_{0}^{1}(\Omega) \times L^{2}(\Omega), \quad \mathscr{H}_{1}=\left(H^{2}(\Omega) \cap H_{0}^{1}(\Omega)\right) \times H_{0}^{1}(\Omega)$, and $\mathscr{H}_{2}=\left(H^{3}(\Omega) \cap H_{0}^{1}(\Omega)\right) \times\left(H^{2}(\Omega) \cap H_{0}^{1}(\Omega)\right)$.

Dissipative wave equations arise as an evolutionary mathematical model in various systems for the relevant physical application including electrodynamics, quantum mechanics, and nonlinear elasticity (see e.g., [1]). The longtime behavior of problem (1) is of a big permanent interest (see e.g. [2-5]), which depends strongly on the growth rate of the nonlinearity $f$. For a long time, in a bounded smooth domain of $\mathbb{R}^{3}$, the global well-posedness for problem (1) holds only in the case of subcubic or cubic nonlinearity (that is, the exponent $p$ in 1.2 less or equals to 3 ), in which the uniqueness is verified by the technology of Sobolev embedding in general. Therefore, the cubic growth rate of nonlinearity had been considered as a critical one for the case of 3-D bounded domain. Consequently, the existence of global attractors for the weakly damped wave equations in the natural energy space $\mathscr{H}$ as well as its regularities only had been known for the case $p \leq 3$, that is, the nonlinear term $f$ at most can be cubic growth in 3 -D bounded domain (see $[2,3,5,6]$ and the references therein).

The case of supercubic growth rate is a bit more complicated since the uniqueness of energy weak solutions is unknown for the supercubic case (e.g., see [7]). However, this problem can be overcome using slightly more regular solutions than the energy ones. These solutions are the so-called Shatah-Struwe solutions. Recently, with the help of the suitable versions of Strichartz estimates, the global well-posedness of quintic wave equation in terms of Shatah-Struwe solutions in 3-D smooth bounded domains has been obtained in [8-10]. Based on the fact that the Strichartz estimates can be 
generalized to weakly damped wave equation, Kalantarov et al. [11] obtained the well-posedness of weakly damped wave equation in the case of quintic nonlinearity in bounded domain of $\mathbb{R}^{3}$, where the solution is the weak solution with extra regularity named Shatah-Struwe solution; moreover, they also considered the global attractor for the case of quintic nonlinearity.

Considering the strong solution, in 1983, Babin and Vishik [3] investigated the damped subcubic wave equation in $\mathscr{H}$. For seeking a compact absorbing set in $\mathscr{H}$, they requested the initial data belonging to $\mathscr{H}_{1}$, and they proved the existence of the maximal $\left(\mathscr{H}_{1}, \mathscr{H}\right)$ attractor for problem (1). Then, Ladyzhenskaya [12] obtained the $\mathscr{H}_{1}$ attractor for cubic nonlinearity. In [13], the second author verified the $\mathscr{H}_{1}$ attractor via a different approach. Recently, the authors [14] have obtained the strong global attractor for subquintic nonlinearity.

As mentioned by many authors, the global attractor, however, does not provide an actual control of the convergence rate of trajectories and it can be sensitive to perturbations. In order to overcome these difficulties, Eden et al. introduced the notion of exponential attractor (see $[15,16])$. In contrary to the global attractor, the exponential attractor is not unique, and it is only semiinvariant. However, it has the advantage of being stable with respect to perturbations, attracts the trajectories exponentially, and has finite fractal dimension. For the strongly damped wave equation, in the subcritical case, Pata and Squassina [17] established an exponential attractor of optimal regularity by using the bootstrap argument; in the critical case, Sun and Yang [18, 19] obtained the exponential attractors based on some asymptotic regularity of the solution. For the weakly damped wave equation, Eden et al. [20] and Grasselli and Pata [21] investigated the exponential attractor for problem (1) with cubic nonlinearity in $\mathscr{H}_{1}$ and $\mathscr{H}$, respectively. For the supercubic nonlinearity, in [22], we established the exponential attractor in $\mathscr{H}$. However, to the best of our knowledge, there is no result on exponential attractor for problem (1) in $\mathscr{H}_{1}$.

In the present paper, as a continuation of our previous works $[14,22]$, we concentrate on the existence of exponential attractor in the subquintic but supercubic case and establish an exponential attractor in the strong topology space based on the Shatah-Struwe solution.

Our main result can be stated as follows.

Theorem 1.. Let $g \in L^{2}(\Omega)$ and $f$ satisfy (2). Then, the solution semigroup $S(t)$ acting on $\mathscr{H}_{1}$ possesses an exponential attractor $\mathscr{A}_{1}$ which is compact in $\mathscr{H}_{1}$, satisfying the following conditions:

(i) $\mathscr{A}_{1}$ is positive invariant, i.e., $S(t) \mathscr{A}_{1} \subset \mathscr{A}_{1}$ for all $t \geq 0$.

(ii) $\operatorname{dim}_{F} \mathscr{A}_{1}<\infty$, i.e., $\mathscr{A}_{1}$ has finite fractal dimension.

(iii) There exist a monotone increasing function $Q_{1}(\cdot)$ and positive constant $\mu$ such that for any bounded set $B \subset \mathscr{H}_{1}$,

$$
\operatorname{dist}_{\mathscr{H}_{1}}\left(S(t) B, \mathscr{A}_{1}\right) \leq Q_{1}\left(\|B\|_{\mathscr{H}_{1}}\right) e^{-\mu t}, \quad \text { for all } t \geq 0
$$

The above theorem will be proved in Section 3. Before that, the preliminary things, including some notations, the well-posedness, and the global attractor of the system are discussed in Section 2.

Throughout the rest of the paper, denote by $C$ any positive constants which may be different from line to line even in the same line; we also denote the different positive constants by $C_{i}, i \in \mathbb{N}$, for special differentiation.

\section{Well-Posedness and Global Attractor}

Firstly, we recall the definitions about the weak solution and strong solution of problem (1) in [11], which will be used to state our results.

Definition 1 (see [11]). For any $T>0$, a function $u(t)$ is a weak solution of problem $(1)$ if $\left(u, u_{t}\right) \in L^{\infty}(0, T ; \mathscr{H})$ and equation (1) is satisfied in the sense of distribution, i.e.,

$$
\begin{gathered}
-\int_{0}^{T}\left(u_{t}, \phi_{t}\right) \mathrm{d} t+\gamma \int_{0}^{T}\left(u_{t}, \phi\right) \mathrm{d} t+\int_{0}^{T}(\nabla u, \nabla \phi) \mathrm{d} t \\
+\int_{0}^{T}(f(u), \phi) \mathrm{d} t=\int_{0}^{T}(g, \phi) \mathrm{d} t,
\end{gathered}
$$

for any $\phi \in C_{0}^{\infty}((0, T) \times \Omega)$. Furthermore, $u(t)$ is a strong solution if $\left(u, u_{t}\right) \in L^{\infty}\left(0, T ; \mathscr{H}_{1}\right)$.

For the well-posedness of strong solution, we have the following result.

Theorem 2 (see [14]). Let $g \in L^{2}(\Omega)$ and $f$ satisfy (2). Then, for every $\left(u_{0}, u_{1}\right) \in \mathscr{H}_{1}$, there exists a unique global strong solution $u(t)$ of problem (1) with energy estimate

$$
\left\|\left(u(t), u_{t}(t)\right)\right\|_{\mathscr{H}_{1}} \leq Q\left(\left\|\left(u_{0}, u_{1}\right)\right\|_{\mathscr{H}_{1}},\|g\|\right)
$$

where monotone function $Q$ is independent of $u$ and $t$. Moreover, for any two strong solutions $u_{1}(t)$ and $u_{2}(t)$, there exists a positive constant $C_{1}$ depending on the $\mathscr{H}_{1}$-norm of $u_{1}(t)$ and $u_{2}(t)$ such that

$$
\left\|\left(\bar{u}(t), \bar{u}_{t}(t)\right)\right\|_{\mathscr{H}_{1}} \leq e^{C_{1} T}\left\|\left(\bar{u}(0), \bar{u}_{t}(0)\right)\right\|_{\mathscr{H}_{1}}, \quad t \in[0, T],
$$

where $\bar{u}(t)=u_{1}(t)-u_{2}(t)$.

Therefore, the strong solution generates a $C_{0}$ operator semigroup $\{S(t)\}_{t \geq 0}$ in $\mathscr{H}_{1}$ as follows:

$$
S(t)\left(u_{0}, u_{1}\right)=\left(u(t), u_{t}(t)\right)
$$

which is continuous in $\mathscr{H}_{1}$.

Finally, we state the existence of global attractor in strong energy space which will be used in Lemma 3.

Theorem 3 (see [14]). Let $g \in L^{2}(\Omega)$ and $f$ satisfy (2). Then, the solution semigroup $S(t)$ associated with problem (1) possesses a global attractor $\mathscr{A}$ in the space $\mathscr{H}_{1}$. 


\section{Proof of Theorem 1}

There are two different approaches to find exponential attractors. The original one (cf. $[15,16])$ relies on the proof that the semigroup $S(t)$ satisfies squeezing property, and the following approach (cf. $[16,17,23]$ ) is established via decomposition of semigroup, which is easier to verify for damped wave equation.

Lemma 1. Let $\mathscr{X}_{0}$ and $\mathscr{X}_{1}$ be two Banach spaces such that $X_{1}$ is compactly embedded into $X_{0}$ and $\{S(t)\}_{t \geq 0}$ is a semigroup on $\mathscr{X}_{0}$. Assume that there exists a bounded positively invariant $\mathscr{X} \subset \mathscr{X}_{0}$ and a time $t_{*}>0$ such that the following hold:

(i) The map

$$
\left(t, z_{0}\right) \longmapsto S(t) z_{0}:\left[0, t_{*}\right] \times \mathscr{X} \longrightarrow \mathscr{X}
$$

is Lipschitz continuous (with the metric inherited from $X_{0}$ ).

(ii) The map $S\left(t_{*}\right): \mathscr{X} \longrightarrow \mathscr{X}$ admits a decomposition of the form

$$
\begin{aligned}
& S\left(t_{*}\right)=S_{0}+S_{1}, \\
& S_{0}: X \longrightarrow X_{0}, \\
& S_{1}: X \longrightarrow X_{1},
\end{aligned}
$$

where $S_{0}$ and $S_{1}$ satisfy the conditions

$$
\begin{aligned}
& \left\|S_{0}\left(z_{1}\right)-S_{0}\left(z_{2}\right)\right\|_{\mathscr{X}_{0}} \leq \frac{1}{4}\left\|z_{1}-z_{0}\right\|_{\mathscr{X}_{0}}, \quad \forall z_{1}, z_{2} \in \mathscr{X}, \\
& \left\|S_{1}\left(z_{1}\right)-S_{1}\left(z_{2}\right)\right\|_{\mathscr{X}_{1}} \leq C_{*}\left\|z_{1}-z_{2}\right\|_{\mathscr{X}_{0}}, \quad \forall z_{1}, z_{2} \in \mathscr{X},
\end{aligned}
$$

for some $C_{*}>0$.

Then, the local dynamical system $(\mathscr{X}, S(t))$ possesses an exponential attractor.

In order to verify the local exponential attractor in $\mathscr{X}$ is an exponential attractor in $\mathscr{H}_{1}$, the following transitivity of exponential attraction is necessary.

Lemma 2 (see [24]). Let $(\mathscr{M}, d)$ be a metric space and $S(t)$ be a semigroup acting on this space such that

$$
d\left(S(t) m_{1}, S(t) m_{2}\right) \leq L_{0} e^{\theta_{0} t} d\left(m_{1}, m_{2}\right),
$$

for some nonnegative constants $L_{0}$ and $\theta_{0}$. Furthermore, we assume that there exist three subsets $M_{1}, M_{2}, M_{3} \subset M$ such that

$$
\begin{aligned}
& \operatorname{dist}_{\mathscr{M}}\left(S(t) M_{1}, M_{2}\right) \leq L_{1} e^{-\theta_{1} t}, \\
& \operatorname{dist}_{\mathscr{M}}\left(S(t) M_{2}, M_{3}\right) \leq L_{2} e^{-\theta_{2} t},
\end{aligned}
$$

for some $\theta_{1}, \theta_{2}>0$ and $L_{1}, L_{2} \geq 0$. Then it follows that

$$
\operatorname{dist}_{\mathscr{M}}\left(S(t) M_{1}, M_{3}\right) \leq L e^{-\theta t},
$$

where $L=L_{0} L_{1}+L_{2}$ and $\theta=\left(\theta_{1} \theta_{2} /\left(\theta_{0}+\theta_{1}+\theta_{2}\right)\right)$.

We will verify all conditions of Lemma 1 step by step.

Step 1. Construct a bounded positively invariant set.

Decompose the solution $u(t)$ as

$$
u(t)=-\Delta^{-1} g+v(t)+w(t),
$$

where $v(t)$ solves the linear problem

$$
\left\{\begin{array}{l}
v_{t t}+\gamma v_{t}-\Delta v=0, \\
v(0)=u_{0}+\Delta^{-1} g, \\
v_{t}(0)=u_{1},
\end{array}\right.
$$

and the remainder $w(t)$ satisfies

$$
\left\{\begin{array}{l}
w_{t t}+\gamma w_{t}-\Delta w=-f(u), \\
w(0)=0 \\
w_{t}(0)=0 .
\end{array}\right.
$$

It is convenient to denote

$$
\begin{aligned}
S_{v}(t) z & =\left(v(t), v_{t}(t)\right), \\
S_{w}(t) z & =\left(w(t), w_{t}(t)\right),
\end{aligned}
$$

where $z=\left(u_{0}, u_{1}\right)$. For equation (15), it is easy to check that the solution is exponentially decaying, namely,

$$
\left\|S_{v}(t) z\right\|_{\mathscr{H}_{1}} \leq C_{3} e^{-\rho t}\left(\|z\|_{\mathscr{H}_{1}}+\|g\|\right)
$$

holds for some $\rho>0$. From [14, Lemma 4.2], we have the following regularity estimate for $S_{w}(t)$ :

$$
\left\|S_{w}(t) z\right\|_{\mathscr{H}_{2}} \leq C \int_{0}^{t} e^{\alpha(s-t)}\|f(u(s))\|_{H^{2}} \mathrm{~d} s \leq C_{4}\left(\|z\|_{\mathscr{H}_{1}},\|g\|\right),
$$

with $\alpha>0$ small enough.

Lemma 3. Let the assumptions in Theorem 1 hold. Then, there exist a compact positive invariant set $\mathscr{B}_{1}$ in $\mathscr{H}_{1}$, positive constant $\mu$, and a monotone increasing function $Q_{2}(\cdot)$ such that for any bounded set $B \subset \mathscr{H}_{1}$,

$$
\operatorname{dist}_{\mathscr{H}_{1}}\left(S(t) B, \mathscr{B}_{1}\right) \leq Q_{2}\left(\|B\|_{\mathscr{H}_{1}}\right) e^{-\mu t} \quad \text { for all } t \geq 0 .
$$

Moreover, the set $\mathscr{B}_{1}+\left(\Delta^{-1} g, 0\right)$ is bounded in $\mathscr{H}_{2}$.

Proof. After vanishing the $v$-component, we can obtain a "perturbed positive trajectory." And the original is asymptotically close to positive trajectory exponentially. Therefore, a collection of perturbed positive trajectories will be a promising candidate for an exponentially attracting set. To carry out this idea, denote $\mathscr{K}_{0}=\left(-\Delta^{-1} g, 0\right)+{\overline{U_{t \geq 0} S_{w}(t) \mathscr{N}}}^{\mathscr{H}_{1}}$, where $\mathcal{N}=\left\{z \in \mathscr{H}_{1}\right.$ : $\left.\operatorname{dist}_{\mathscr{H}_{1}}(z, \mathscr{A})<1\right\}$ is a neighborhood of the global attractor $\mathscr{A}$. We will show that $\mathscr{K}_{0}$ is an exponentially attracting set. 
Notice that $\mathcal{N}$ is an absorbing set. Hence, for any given bounded set $B \subset \mathscr{H}_{1}$, there exists $T=T\left(\|B\|_{\mathscr{H}_{1}}\right)$ such that $S(t) B \subset \mathcal{N}$, for all $t \geq T$. Furthermore, for $t \geq T$, we have

$$
\begin{aligned}
\operatorname{dist}_{\mathscr{H}_{1}}\left(S(t) B, \mathscr{K}_{0}\right) \leq & \operatorname{dist}_{\mathscr{H}_{1}}\left(S(t-T) S(T) B,\left(-\Delta^{-1} g, 0\right)\right. \\
& \left.+S_{w}(t-T) \mathcal{N}\right) \\
\leq & \operatorname{dist}_{\mathscr{H}_{1}}\left(S(t-T) \mathcal{N},\left(-\Delta^{-1} g, 0\right)\right. \\
& \left.+S_{w}(t-T) \mathcal{N}\right) \\
\leq & \left\|S_{v}(t-T) \mathcal{N}\right\|_{\mathscr{H}_{1}} \leq C_{3} e^{-\rho(t-T)} \\
& \cdot\left(\|\mathcal{N}\|_{\mathscr{H}_{1}}+\|g\|\right) .
\end{aligned}
$$

On the other hand, by inequality (5), we have the uniform estimate

$$
\operatorname{dist}_{\mathscr{H}_{1}}\left(S(t) B, \mathscr{K}_{0}\right) \leq C_{5}, \quad t \geq 0,
$$

for some $C_{5}$ depending only on $\|B\|_{\mathscr{H}_{1}}$. Combining the two above inequalities, we obtain

$$
\operatorname{dist}_{\mathscr{H}_{1}}\left(S(t) B, \mathscr{K}_{0}\right) \leq\left(C_{5} e^{\rho T}+C_{3} e^{\rho T}\left(\|\mathcal{N}\|_{\mathscr{H}_{1}}+\|g\|\right)\right) e^{-\rho t},
$$

Then, we define a positive invariant set by $\mathscr{K}_{1}={\overline{U_{t \geq 0} S(t) \mathscr{K}_{0}}}$, which satisfies

$\operatorname{dist}_{\mathscr{H}_{1}}\left(S(t) B, \mathscr{K}_{1}\right) \leq\left(C_{5}+C_{3} e^{\rho T}\left(\|\mathcal{N}\|_{\mathscr{H}_{1}}+\|g\|\right)\right) e^{-\rho t}, \quad t \geq 0$.

It remains to show that $\mathscr{K}_{1}+\left(\Delta^{-1} g, 0\right)$ is bounded in $\mathscr{H}_{2}$. Inequality (19) implies $S_{w}(t) \mathscr{K}_{0}$ and $\mathscr{K}_{0}+\left(\Delta^{-1} g, 0\right)$ are both bounded in $\mathscr{H}_{2}$. Because $S(t) \mathscr{K}_{0} \subset$ $\left(-\Delta^{-1} g, 0\right)+S_{v}(t) \mathscr{K}_{0}+S_{w}(t) \mathscr{K}_{0}$, we will complete our proof if we can show $S_{v}(t) \mathscr{K}_{0}$ is bounded (uniformly in $t \geq 0$ ) in $\mathscr{H}_{2}$. Note that $S_{v}(t)\left(u_{0}, u_{1}\right)$ is generated by linear problem

$$
\left\{\begin{array}{l}
v_{t t}+\gamma v_{t}-\Delta v=0, \\
v(0)=u_{0}+\Delta^{-1} g, \\
v_{t}(0)=u_{1},
\end{array}\right.
$$

and it is clear that $S_{v}(\cdot)\left(u_{0}, u_{1}\right) \in L^{\infty}\left(\mathbb{R}^{+} ; \mathscr{H}_{2}\right)$ if $\left(u_{0}+\Delta^{-1} g, u_{1}\right) \in \mathscr{H}_{2}$. Thus, $\mathscr{K}_{0}+\left(\Delta^{-1} g, 0\right) \subset \mathscr{H}_{2}$ implies $S_{v}(t) \mathscr{K}_{0}$ is bounded in $\mathscr{H}_{2}$. Therefore, by setting $\mathscr{B}_{1}=\mathscr{K}_{1}$, the proof is completed.

Step 2. We will verify asymptotic regularity and Lipschitz continuity.

Lemma 4. Let the assumptions in Theorem 1 hold. Then, there exists a time $t_{*}>0$ such that the following properties hold:

(i) We have estimates
$\left\|S_{v}\left(t_{*}\right) z_{1}-S_{v}\left(t_{*}\right) z_{2}\right\|_{\mathscr{H}_{1}} \leq \frac{1}{4}\left\|z_{1}-z_{2}\right\|_{\mathscr{H}_{1}}, \quad$ for all $z_{1}, z_{2} \in \mathscr{B}_{1}$,

$\left\|S_{w}\left(t_{*}\right) z_{1}-S_{w}\left(t_{*}\right) z_{2}\right\|_{\mathscr{C}_{2}} \leq K\left\|z_{1}-z_{2}\right\|_{\mathscr{C}_{1}} \quad$ for all $z_{1}, z_{2} \in \mathscr{B}_{1}$,

where the constant $K$ depends only on $t_{*}$ and $\left\|\mathscr{B}_{1}\right\|_{\mathscr{H}_{1}}$.

(ii) The mapping $(t, z) \longmapsto S(t) z$ is Lipschitz continuous in $\mathscr{H}_{1}$-norm on $\left[0, t_{*}\right] \times \mathscr{B}_{1}$.

Proof. (i) For any $z_{1}=\left(u_{0}^{1}, u_{1}^{1}\right), z_{2}=\left(u_{0}^{2}, u_{1}^{2}\right) \in \mathscr{B}_{1}$, set $\left(\bar{v}(t), \bar{v}_{t}(t)\right)=S_{v}(t) z_{1}-S_{v}(t) z_{2} \quad$ and $\quad\left(\bar{w}(t), \bar{w}_{t}(t)\right)=$ $S_{w}(t) z_{1}-S_{w}(t) z_{2}$. Then, $\left(\bar{v}(t), \bar{v}_{t}(t)\right)$ and $\left(\bar{w}(t), \bar{w}_{t}(t)\right)$ solve the following equations, respectively:

$$
\begin{aligned}
& \left\{\begin{array}{l}
\bar{v}_{t t}+\gamma \bar{v}_{t}-\Delta \bar{v}=0, \\
\bar{v}(0)=u_{0}^{1}-u_{0}^{2}, \\
\bar{v}_{t}(0)=u_{1}^{1}-u_{1}^{2},
\end{array}\right. \\
& \left\{\begin{array}{l}
\bar{w}_{t t}+\gamma \bar{w}_{t}-\Delta \bar{w}=f\left(u^{2}\right)-f\left(u^{1}\right), \\
\bar{w}(0)=0, \\
\bar{w}_{t}(0)=0 .
\end{array}\right.
\end{aligned}
$$

From equation (27), we can get exponential decay estimate $\left\|\left(\bar{v}(t), \bar{v}_{t}(t)\right)\right\|_{\mathscr{C}_{1}} \leq C_{3} e^{-\rho t}\left\|z_{1}-z_{2}\right\|_{\mathscr{C}_{1}}$. Hence, by setting $t_{*}=(1 / \rho) \ln \left(4 C_{3}\right)$, we have

$$
\left\|\left(\bar{v}(t), \bar{v}_{t}(t)\right)\right\|_{\mathscr{C}_{1}} \leq \frac{1}{4}\left\|z_{1}-z_{2}\right\|_{\mathscr{C}_{1}} .
$$

Taking inner product of (28) with $\Delta^{2} \bar{w}_{t}$ and integrating on $\left[0, t_{*}\right]$, we can obtain

$$
\left\|\left(\bar{w}(t), \bar{w}_{t}(t)\right)\right\|_{\mathscr{H}_{2}} \leq C\left(\int_{0}^{t_{*}}\left\|f\left(u^{2}(s)\right)-f\left(u^{1}(s)\right)\right\|_{H^{2} \cap H_{0}^{1}}^{2} \mathrm{~d} s\right)^{1 / 2} .
$$

Since

$$
\begin{aligned}
\left\|f\left(u^{2}\right)-f\left(u^{1}\right)\right\|_{H^{2} \cap H_{0}^{1}}^{2}= & \left\|\Delta\left(f\left(u^{2}\right)-f\left(u^{1}\right)\right)\right\| \\
= & \left\|\Delta \int_{0}^{1} f^{\prime}\left(u^{1}-\tau \bar{u}\right) \bar{u} \mathrm{~d} \tau\right\| \\
= & \| \int_{0}^{1}\left(f^{\prime \prime \prime}\left(u^{1}-\tau \bar{u}\right)\left|\nabla\left(u^{1}-\tau \bar{u}\right)\right|^{2} \bar{u}\right. \\
& +2 f^{\prime \prime}\left(u^{1}-\tau \bar{u}\right) \nabla\left(u^{1}-\tau \bar{u}\right) \nabla \bar{u} \\
& \left.+f^{\prime}\left(u^{1}-\tau \bar{u}\right) \Delta \bar{u}\right) \mathrm{d} \tau \| \\
\leq & C\left(1+\left\|u^{1}\right\|_{H^{2}}^{p-1}+\left\|u^{2}\right\|_{H^{2}}^{p-1}\right)\|\Delta \bar{u}\|,
\end{aligned}
$$

we have

$$
\left\|\left(\bar{w}(t), \bar{w}_{t}(t)\right)\right\|_{\mathscr{H}_{2}} \leq C^{*}\left\|z_{1}-z_{2}\right\|_{\mathscr{H}_{1}},
$$


for some $C^{*}$ depending on $\left\|\mathscr{B}_{1}\right\|_{\mathscr{H}_{1}}$ and $t_{*}$.

(ii) For $z_{1}, z_{2} \in \mathscr{B}_{1}$ and $t_{1}, t_{2} \in\left[0, t_{*}\right]$, we have

$$
\begin{aligned}
\left\|S\left(t_{1}\right) z_{1}-S\left(t_{2}\right) z_{2}\right\|_{\mathscr{H}_{1}} \leq & \left\|S\left(t_{1}\right) z_{1}-S\left(t_{1}\right) z_{2}\right\|_{\mathscr{H}_{1}} \\
& +\left\|S\left(t_{1}\right) z_{2}-S\left(t_{2}\right) z_{2}\right\|_{\mathscr{H}_{1}} .
\end{aligned}
$$

The first term is handled by estimate (6). Recall that $\mathscr{B}_{1}$ is invariant, and thus $S(\cdot) \mathscr{B}_{1}+\left(\Delta^{-1} g, 0\right) \in L^{\infty}\left(0, \infty ; \mathscr{H}_{2}\right)$. Substituting the $\mathscr{H}_{2}$ regularity result into equation (1), we have $(\mathrm{d} / \mathrm{d} t) S(\cdot) \mathscr{B}_{1} \in L^{\infty}\left(0, \infty ; \mathscr{H}_{1}\right)$. This yields that for the second term in the right hand of (33), we have

$$
\begin{aligned}
\left\|S\left(t_{1}\right) z_{2}-S\left(t_{2}\right) z_{2}\right\|_{\mathscr{H}_{1}} & \leq\left|\int_{t_{1}}^{t_{2}}\left\|\frac{\mathrm{d}}{\mathrm{d} t} S(s) z_{2}\right\|_{\mathscr{H}_{1}} \mathrm{~d} s\right| \\
& \leq\left\|\frac{\mathrm{d}}{\mathrm{d} t} S(s) z_{2}\right\|_{L^{\infty}\left(0, t_{*} ; \mathscr{H}_{1}\right)}\left|t_{1}-t_{2}\right| .
\end{aligned}
$$

Therefore, applying Lemma 1, we can prove the existence of an exponential attractor $\mathscr{A}_{1}$ for $\{S(t)\}_{t \geq 0}$ in $\mathscr{B}_{1}$ immediately. From the construction of $\mathscr{B}_{1}, \mathscr{B}_{1}$ exponentially attracts all bounded subsets of the whole phase-space $\mathscr{H}_{1}$. Then, the transitivity property of exponential attraction implies that $\mathscr{A}_{1}$ attracts all bounded subsets of the whole phase-space $\mathscr{H}_{1}$ exponentially, i.e., $\mathscr{A}_{1}$ is an exponential attractor in $\mathscr{H}_{1}$.

\section{Data Availability}

The data used to support the study are included within the article.

\section{Conflicts of Interest}

The authors declare that they have no conflicts of interest.

\section{Acknowledgments}

C. Liu was supported by the Natural Science Foundation of Jiangsu Province (Grant no. BK20170308) and NSFC (11801227); F. Meng was supported by NSFC (11701230); C. Zhang was supported by NSFC (11801228) and the dual creative (innovative and entrepreneurial) talents project in Jiangsu Province.

\section{References}

[1] V. V. Chepyzhov and M. I. Vishik, Attractors for Equations of Mathematical Physics, American Mathematical Society, Providence, RI, USA, 2002.

[2] J. Arrieta, A. N. Carvalho, and J. K. Hale, "A damped hyerbolic equation with critical exponent," Communications in Partial Differential Equations, vol. 17, no. 5-6, pp. 841-866, 1992.

[3] A. V. Babin and M. I. Vishik, "Regular attractors of semigroups and evolution equation," Journal de Mathématiques Pures et Appliquées, vol. 62, pp. 441-491, 1983.

[4] A. V. Babin and M. I. Vishik, Attractors of Evolution Equations, North-Holland, Amsterdam, Netherlands, 1992.
[5] V. Pata, S. Zelik, and S. Zelik, "A remark on the damped wave equation," Communications on Pure \& Applied Analysis, vol. 5, no. 3, pp. 611-616, 2006.

[6] R. Temam, "Infinite-dimensional dynamical systems in mechanics and physics," Applied Mathematical Sciences, Vol. 68, Springer-Verlag, New York, NY, USA, 2nd edition, 1997.

[7] J. Ball, "Global attractors for damped semilinear wave equations," Discrete \& Continuous Dynamical Systems, vol. 10, pp. 31-52, 2004.

[8] M. D. Blair, H. F. Smith, and C. D. Sogge, "Strichartz estimates for the wave equation on manifolds with boundary," Annales de l'Institut Henri Poincare (C) Non Linear Analysis, vol. 26, no. 5, pp. 1817-1829, 2009.

[9] N. Burq, G. Lebeau, and F. Planchon, "Global existence for energy critical waves in 3D domains," Journal of the American Mathematical Society, vol. 21, no. 3, p. 831, 2008.

[10] N. Burq and F. Planchon, "Global existence for energy critical waves in 3-D domains: Neumann boundary conditions," American Journal of Mathematics, vol. 131, pp. 1715-1742, 2009.

[11] V. Kalantarov, A. Savostianov, and S. Zelik, "Attractors for damped quintic wave equations in bounded domains," Annales Henri Poincaré, vol. 17, no. 9, pp. 2555-2584, 2016.

[12] O. A. Ladyzhenskaya, "Attractors of nonlinear evolution problems with dissipation," Journal of Soviet Mathematics, vol. 40, no. 5, pp. 632-640, 1988.

[13] F. Meng, "Strong global attractors for 3D wave equations with weakly damping," Abstract and Applied Analysis, vol. 2012, Article ID 469382, 12 pages, 2012.

[14] C. Liu, F. Meng, and C. Zhang, "Strong global attractor for weakly damped wave equation with sub-quintic nonlinearity," Applied Mathematics Letters, vol. 98, pp. 314-321, 2019.

[15] A. Eden, C. Foias, B. Nicolaenko, and R. Temam, "Ensembles inertiels pour des équations d'évolution dissipatives," Comptes Rendus de l'Académie des Sciences Paris, vol. 310, pp. 559-562, 1990.

[16] A. Eden, C. Foias, B. Nicolaenko, and R. Temam, Exponential Attractors for Dissipative Evolution Equations, Masson, Paris, France, 1994.

[17] V. Pata and M. Squassina, "On the strongly damped wave equation," Communications in Mathematical Physics, vol. 253, no. 3, pp. 511-533, 2005.

[18] C. Sun, "Asymptotic regularity for some dissipative equations," Journal of Differential Equations, vol. 248, no. 2, pp. 342-362, 2010.

[19] M. Yang and C. Sun, "Exponential attractors for the strongly damped wave equations," Nonlinear Analysis: Real World Applications, vol. 11, no. 2, pp. 913-919, 2010.

[20] A. Eden, A. J. Milani, and B. Nicolaenko, "Finite dimensional exponential attractors for semilinear wave equations with damping," Journal of Mathematical Analysis and Applications, vol. 169, no. 2, pp. 408-419, 1992.

[21] M. Grasselli and V. Pata, "On the damped semilinear wave equation with critical exponent," in Proceedings of the 4th Internatinal Conference on Dynamical Systems and Differential Equations, pp. 351-358, Wilmington, NC, USA, May 2002.

[22] F. Meng and C. Liu, "Exponential attractors for weakly damped wave equation with sub-quintic nonlinearity," Computers \& Mathematics with Applications, vol. 78, no. 3, pp. 1026-1036, 2019.

[23] M. Efendiev, S. Zelik, and A. Miranville, "Exponential attractors and finite-dimensional reduction for non-autonomous dynamical systems," Proceedings of the Royal Society of 
Edinburgh: Section A Mathematics, vol. 135, no. 4, pp. 703730, 2005.

[24] P. Fabrie, C. Galusinski, A. Miranville, and S. V. Zelik, "Uniform exponential attractors for a singularly perturbed damped wave equation," Discrete \& Continuous Dynamical Systems, vol. 10, pp. 211-238, 2004.

[25] A. N. Carvalho, J. W. Cholewa, and T. Dlotko, "Damped wave equations with fast growing dissipative nonlinearites," Discrete \& Continuous Dynamical Systems, vol. 24, pp. 1147$1165,2009$.

[26] J. K. Hale, Asymptotic Behavior of Dissipative Systems, American Mathematical Society, Providence, RI, USA, 1988.

[27] J. Lions, Quelques Méthodes de Résolution des Problèmes aux Limites n-Linéaires, Dunod, Paris, France, 1969.

[28] J. Shatah and M. Struwe, "Well-posedness in the energy space for semilinear wave equations with critical growth," International Mathematics Research Notices, vol. 1994, no. 7, pp. 303-309, 1994.

[29] H. Triebel, Interpolation Theory, Function Spaces, Differential Operators, North-Holland Publishing Company, Amsterdam, Netherlands, 1978.

[30] S. Zelik, "Asymptotic regularity of solutions of singularly perturbed damped wave equations with supercritical nonlinearities," Discrete \& Continuous Dynamical Systems, vol. 11, no. 2-3, pp. 351-392, 2004. 